\title{
FUSION OF TERRASAR-X AND RAPIDEYE DATA: A QUALITY ANALYSIS
}

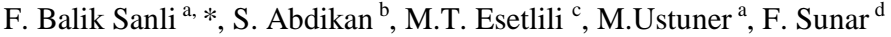 \\ ${ }^{a}$ Yildiz Technical University, Civil Engineering Faculty, Department of Geomatic Engineering, 34220 Esenler-Istanbul, \\ Turkey - fbalik@yildiz.edu.tr, mustuner@yildiz.edu.tr \\ ${ }^{b} B e e S e n s e$, Consultancy and Engineering on Geo-information Technologies, 2628 VG, Delft, Netherlands - \\ s.abdikan@beesense.nl \\ ${ }^{c}$ Ege University, Faculty of Agriculture,Deparment of Soil Science and Plant Nutrition, \\ 35100 Bornova-İzmir/ Turkey - tolga.esetlili@ege.edu.tr \\ ${ }^{d}$ Istanbul Technical University, Civil Engineering Faculty, Department of Geomatic Engineering, 34469 Maslak- \\ Istanbul,Turkey - fsunar@itu.edu.tr
}

\section{Commission VII, WG 6}

KEY WORDS: Image fusion, TerraSAR-X, RapidEye, Multi-sensor

\begin{abstract}
:
This research compares and evaluates image fusion algorithms to achieve spatially improved images while preserving the spectral information. In order to compare the performance of fusion techniques both active and passive images were used. As an active image a high resolution, X-band, VV polarized TerraSAR-X data and as a multispectral image RapidEye data were used. RapidEye provides five optical bands in the 400-850 $\mathrm{nm}$ range and it is the first space-borne sensor which operationally gathers the red edge spectrum (690-730 $\mathrm{nm}$ ) besides the standard channels of multi-spectral satellite sensors. The selected study area is in the low lands of Menemen (Izmir) Plain on the west of Gediz Basin covering both agricultural fields and residential areas. For the quality analysis, Adjustable SAR-MS Fusion (ASMF), Ehlers fusion and High Pass Filtering (HPF) approaches were investigated. In this study preliminary results of selected image fusion methods were given. The quality of the fused images was assessed with qualitative and quantitative analyses. For the qualitative analysis visual comparison was applied using different band combinations of fused image and original multispectral Rapid-Eye image. In the merged images color distortions regarding to SAR-optical synergy were investigated. Statistical analysis was carried out as quantitative analyses. In this respect Correlation Coefficient (CC), Standard Deviation Difference (SDD), Universal Image Quality Index (UIQI) and Root Mean Square Error (RMSE) were performed for quality assessments. In general HPF was performed best while ASMF was performed the worst in all results.
\end{abstract}

\section{INTRODUCTION}

A variety of remote sensing satellites provide data for the observation of phenomena on Earth. For many mapping purposes both high spatial resolution and high spectral resolution are essential. Data fusion is a common method to integrate spatial and spectral information for a better interpretation and analysis (Abdikan and Sanli, 2012).

Conventionally, high resolution Panchromatic (PAN) image is fused with low resolution Multispectral (MS) image which are synchronously acquired from same satellite (Yıldırım and Güngör, 2012; Witharana et al, 2013). Moreover, using PAN and MS images from different satellites were also applied successfully (Ehlers et al, 2010; Jalan et al, 2012). Not only optical and optical image fusion but also optical and synthetic aperture radar (SAR) image fusion has been chosen for some researches (Ehlers et al, 2010; Abdikan and Sanli, 2012).

Data fusion is a challenging application. Many approaches have been proposed and several comparison analyses were studied to find out the most ideal method. However, for different data sets and applications variable results have been achieved.
Klous (2008) indicated that for the fusion of Quickbird and TerraSAR-X data without color distortions only the Ehlers and the Additive Wavelet Luminance Proportional (AWLP) are useful among the eight methods namely AWLP, Brovey, CN, Ehlers, GST, MIHS, PCA and UNB. But spatial resolution was only improved with Ehlers method.

Berger et al (2010) fused high resolution spotlight mode TerraSAR-X and RapidEye for a statistical comparison using Principal Component Substitution (PCS), Ehlers method, GST, HPF, MIHS and wavelet algorithms. In that study, according to visual performance only Ehlers and HPF gave better results. In statistics mean bias, difference in variances, standard deviation of the difference image, CC, SAM, ERGAS, spatial CC and profile intensity curve quality metrics were measured. All measures gave different results, for the evaluation mean of all measures were calculated and ranked. The authors planned to analyse potential of HPF method to improve the urban land cover classification in their future studies. Ehlers method achieved second best performance among the all fusion methods.

Ehlers et al (2010) evaluated PC, CN, GST, UNB and Ehlers methods using both optical-optical and optical-SAR pairs. It is showed that TerraSAR-X data could be useful to improve the

\footnotetext{
* Corresponding author: Email: fbalik@yildiz.edu.tr
} 
spatial resolution of MS data. Among these techniques only Ehlers preserved the spectral information of MS data, and the rest of them were suggested to fuse single sensor and single date images.

In the study area, previous research showed that HPF and Wavelet methods were resulting similar in both RADARSATSPOT and PALSAR-SPOT fused images. However, HPF method using PALSAR data gave the best result for agricultural areas (Abdikan et al 2008). This study extended by adding Ehlers method and analysing all in two different regions (i.e. urban and agricultural) (Abdikan and Sanli 2012). In that research among the five methods used, Ehlers preserved spectral quality better than other four methods (IHS, PCA, Wavelet and HPF). In addition to that, different combination of SAR-SAR and optical-SAR fusion, using RADARSAT, PALSAR, ENVISAT and SPOT images, were applied with IHS, Brovey and Ehlers methods (Abdikan et al., 2012). Visual and statistical evaluations demonstrated that Ehlers fusion had a better result.

In this study it is aimed to make a comparative quality analysis of image fusion done using TerraSAR-X and RapidEye data. The contribution of SAR data to RapidEye over the agricultural land types is under investigation.

\section{STUDY AREA AND MATERIALS}

\subsection{Test Site}

The study area is located on the west cost of Turkey in the Menemen Plain of Izmir Province. On the west of area Aegean Sea lies and on the south bordered with Izmir Bay (Figure 1). The area which is approximately $50 \mathrm{~km}^{2}$, mostly covers agricultural fields depending on the harvesting period and crop species. On the date of satellite data acquisition, the fields were covered with summer crops such as corn, cotton, water-melon and meadow. There are also residential areas and small water bodies in the area. The topographic relief of the study area is lower than $1 \%$.

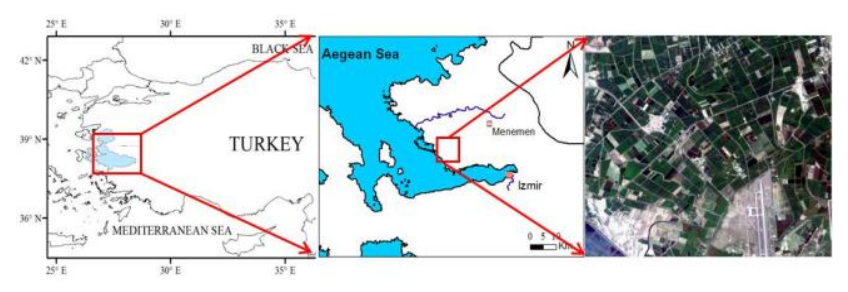

Figure 1. The study area: Menemen Plain

\subsection{Dataset}

In the analysis, a high resolution TerraSAR-X SAR data and multispectral RapidEye data were used. TerraSAR-X image was acquired in ascending pass and pre-processed as Enhanced Earth Corrected (EEC) type (i.e. radiometrically enhanced). Data was acquired in Strip Map (SM) mode with stripNear_011 and dual polarization. In this paper only VV polarization data was used in the analysis. The specifications of the data used are given in Table 1.

The Rapideye image was acquired as L3A format, which is radiometrically calibrated and orthorectified data and resampled to $5 \times 5 \mathrm{~m}$ ground resolution.

\begin{tabular}{|l|c|c|}
\hline & TERRASAR-X & RAPIDEYE \\
\hline Date & 29.08 .2010 & 10.08 .2010 \\
Ground & $8 \times 8$ & $5 \times 5$ \\
Resolution (m) & & $440-510 \mathrm{~nm}$ (Blue) \\
& & $520-590 \mathrm{~nm}$ (Green) \\
Wavelength & $5.6 \mathrm{~cm}$ & $630-685 \mathrm{~nm}$ (Red) \\
& (X-band) & $690-730 \mathrm{~nm}$ (Red Edge) \\
& & $760-850 \mathrm{~nm}$ ( NIR) \\
Polarization & VV/VH & - \\
Incidence Angle & $38.06-39.37$ & 11.38 \\
\hline
\end{tabular}

Table 1. Specifications of dataset

\section{METHODOLOGY}

\subsection{Pre-processing}

Before the application of image fusion multiple steps of image pre-processing are necessary. The first step is filtering to reduce speckles in SAR image. Therefore, a gamma map filtering with $3 \times 3$ kernel window was chosen. Afterward TerraSAR-X image was registered to RapidEye image with a less than \pm 1 pixel root mean square error and resampled to its original pixel size as $8 \times 8$ $\mathrm{m}$.

\subsection{Image Fusion Methods}

In the study, three different pixel level image fusion approaches have been examined. These are Adjustable SAR-MS Fusion (ASMF), Ehlers fusion and High Pass Filtering (HPF).

ASMF is able to fuse both high resolution SAR data with low resolution MS data, and low resolution SAR data with high resolution MS data. It stretches the MS grey level from the MS grey value to the range of zero to SAR grey value range. Scaling values are specified for each SAR and MS images (PCI Geomatica, 2012; Zhang 2009). This method gives user the opportunity of weighting both SAR and optical image. To investigate the effects of weighting of different data sets, two images were created namely ASMF-I and ASMF-II. In ASMF-I both SAR and MS images were weighted $100 \%$, while in ASMF-II SAR and MS were weighted $50 \%$ and $100 \%$ respectively.

As a hybrid technique the Ehlers fusion uses IHS method with a Fourier domain filtering. Low-pass filter is used for the intensity spectrum and high-pass filter is used for panchromatic image. It preserves spectral characteristics of optical image (Ehlers et al, 2010).

HPF method fuses spatial and spectral information with a band addition approach. First high resolution image is filtered with a high-pass filter. Then filtered image was added to each low resolution multispectral image ( $\mathrm{Lu}$ et al, 2011; Abdikan and Sanli 2012). 


\subsection{Quality Analysis}

Fusion quality assessment was conducted regarding to (1) visual comparison and (2) statistical analysis. As a qualitative analysis visual comparison was performed between fused images and the RapidEye image to test the color distortions. Quantitative analysis was applied using different quality metrics such as Correlation Coefficient (CC), Standard Deviation Difference (SDD), Universal Image Quality Index (UIQI) and Root Mean Square Error (RMSE).

CC shows the correlation between the original image and the fused image. The closest to the value 1 gives the better correlation. SDD is the difference of standard deviation values relative to the mean of the original MS data. It is expected to be close to the zero. UIQI has been designed using combination of luminance distortion, contrast distortion and loss of correlation (Wang and Bovik, 2002). The highest UIQI value indicates the best spectral quality. RMSE was computed from mean and standard deviation values of both MS and fused images (Hong et al, 2009). For a better correspondence between original MS and fused images RMSE value should be low.

\section{RESULTS}

\subsection{Visual Evaluation}

In the visual analysis, a subset from the study area (mostly cotton fields seen as orange and corn fields seen as red shown in Figure 2a) was selected and fused image outputs were compared with the original MS Rapideye image. A false color composite ( $\mathrm{R}=\mathrm{NIR}, \mathrm{G}=\mathrm{Red}$ Edge, $\mathrm{B}=\mathrm{Red}$ ) Rapideye image was used as a reference image (Figure 2). The fused images of Ehlers and HPF have the resolution of $5 \mathrm{~m}$ while the results of ASMF have $8 \mathrm{~m}$ resolution. Before comparing with MS image ASMF images were resampled to the resolution of the original MS.

As can be seen clearly, the HPF method has the most similar result which preserves the color in the original data (Figure 2c). The contribution of SAR data can be visible with some roughness at agricultural areas. Furthermore, it is also the best technique which provides the spatial improvement.

Ehlers method preserves color information but also introduces blurriness which was noticed in some parts of fields (Figure 2d). Meanwhile, there are some loses in details especially on linear features such as borders of fields. It could be considered the worst method in terms of contribution of SAR.

In general ASMF method has the poor result on visual comparisons. ASMF-I has the worst results with color distortions (Figure 2e). The weighting of SAR image gave different results. If the weighting of SAR is diminished, spectral information could be better preserved (Figure 2f). However, the contribution of SAR data might be less visible (Figure 2e-2f). Eventually, ASMF-II gave a better spectral preservation than ASMF-I. When assessing the spatial information, linear details were similar in both ASMF results.

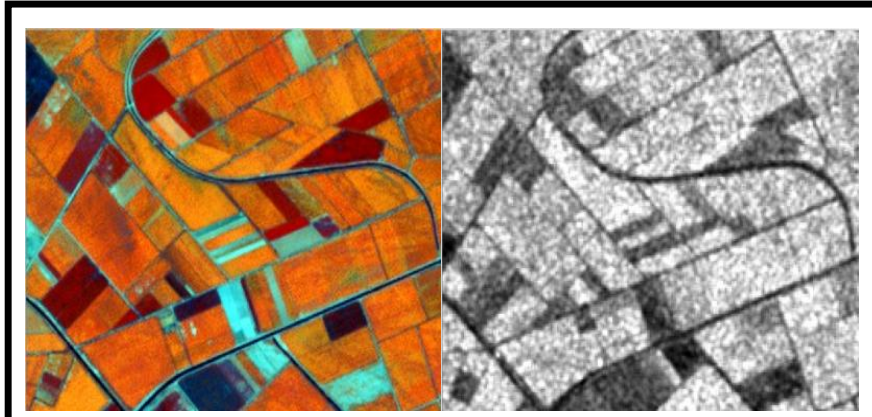

a-) RapidEye $(5,4,3)$

b-) TerraSAR-X

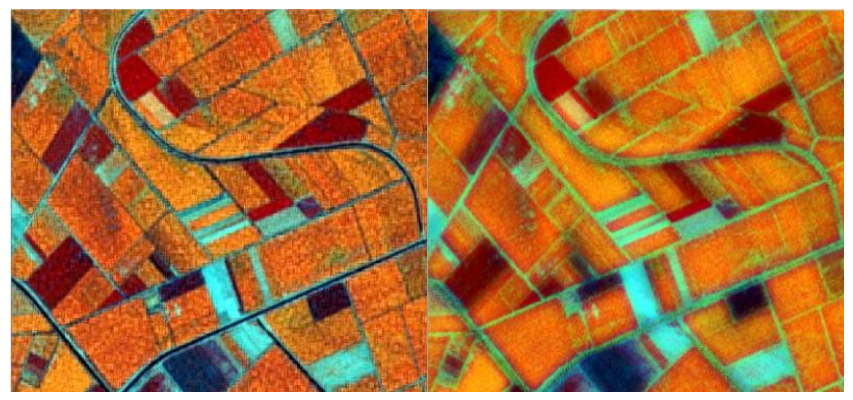

c-) HPF

d-) Ehlers

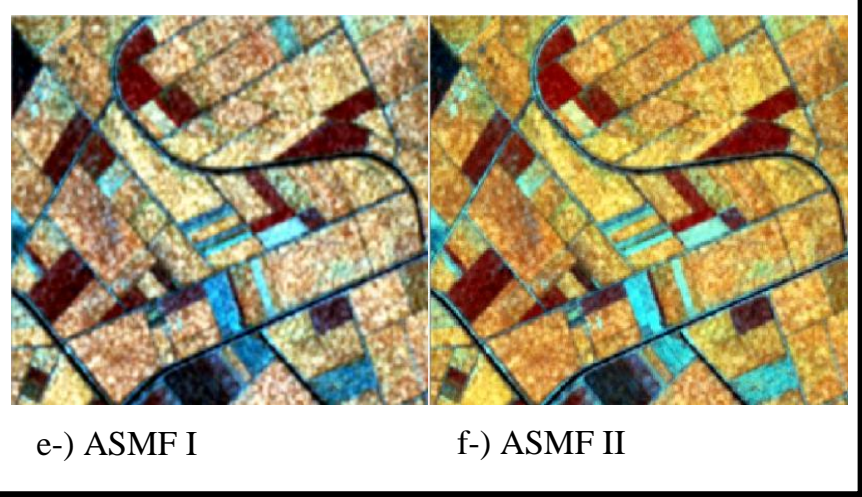

Figure 2. Comparison of fused images with RapidEye data

\subsection{Statistical Evaluation}

Quantitative analyses were applied to verify the qualitative assessment using four quality metrics namely, SDD, CC, UIQI and RMSE (Table 2).

All algorithms demonstrated the similar performance for SDD which are close to zero. Although HPF and Ehlers results have smaller SDD values, Ehlers has better results for three of five bands. Following the HPF ASMF-II ranks in third order. In the other three metrics (CC, UIQI, RMSE), HPF performed the best among the all methods. All bands of HPF fused image has significantly higher $\mathrm{CC}$ values which are higher than 0,96 . In second order, Ehlers fused image has considerable $\mathrm{CC}$ values with an approximate average of 0,89. ASMF-II follows Ehlers with quite high values and, lastly ASMF-I comes with the lowest $\mathrm{CC}$ values.

HPF results of UIQI also indicate high values which is the top among the all results. Here again Ehlers was in the second order and ASMF-I have had the worst performance. The RMSE values gave a noticeable difference between HPF and the rest. 
Consequently, the quality metrics of HPF was superior to the other methods. Furthermore, the ASMF-I fused result reported the poorest values in all metrics. Ehlers gave satisfactory results in SDD, CC and UIQI. ASMF-II also indicates high records in SDD and CC metrics.

\begin{tabular}{|c|c|c|c|c|c|}
\hline & & SDD & $\mathrm{CC}$ & UIQI & RMSE \\
\hline \multirow{5}{*}{ HPF } & Blue & -0.0056 & 0.9692 & 0.9681 & 226.1466 \\
\hline & Green & -0.0038 & 0.9715 & 0.9712 & 257.1184 \\
\hline & Red & 0.0018 & 0.9745 & 0.9744 & 374.0140 \\
\hline & RedEdge & -0.0038 & 0.9708 & 0.9705 & 222.9353 \\
\hline & NIR & 0.0017 & 0.9774 & 0.9774 & 650.7761 \\
\hline \multirow{5}{*}{ Ehlers } & Blue & 0.0044 & 0.7730 & 0.6835 & 5079.1058 \\
\hline & Green & 0.0054 & 0.8564 & 0.8482 & 1138.2881 \\
\hline & Red & 0.0017 & 0.9599 & 0.9598 & 470.0827 \\
\hline & RedEdge & 0.0108 & 0.8728 & 0.8311 & 2095.5694 \\
\hline & NIR & 0.0008 & 0.9687 & 0.9686 & 784.9260 \\
\hline \multirow{5}{*}{$\begin{array}{c}\text { ASMF } \\
\text { I }\end{array}$} & Blue & 0.0406 & 0.4236 & 0.2025 & 5563.7432 \\
\hline & Green & 0.0726 & 0.3659 & 0.1983 & 4418.9484 \\
\hline & Red & 0.2415 & 0.3657 & 0.1642 & 2863.4821 \\
\hline & RedEdge & 0.0442 & 0.4560 & 0.3715 & 2605.4231 \\
\hline & NIR & 0.1753 & 0.9271 & 0.3204 & 8225.9421 \\
\hline \multirow{5}{*}{$\begin{array}{c}\text { ASMF } \\
\text { II }\end{array}$} & Blue & -0.0045 & 0.7785 & 0.4654 & 5168.0117 \\
\hline & Green & 0.0252 & 0.7718 & 0.5914 & 3649.3621 \\
\hline & Red & 0.1661 & 0.8041 & 0.5775 & 2246.7963 \\
\hline & RedEdge & 0.0092 & 0.7128 & 0.6858 & 1515.7211 \\
\hline & NIR & 0.1458 & 0.9519 & 0.5000 & 7046.1106 \\
\hline
\end{tabular}

Table 2. Results of quality metrics

\section{CONCLUSIONS}

In this study, performance of three fusion methods was investigated over agricultural fields. For the fusion processes five-band RapidEye and VV polarized TerraSAR-X images were used. The results indicated that the HPF fused image conserved the spectral characteristics in the original RapidEye data and also improved the spatial information. Following the HPF, Ehlers method comes in the second order. Although having higher quality metrics, it introduced color distortions. Both ASMF I and II fused images presented the worse results in visual comparison. However, ASMF-II performed better than ASMF-I in terms of spectral preservation and statistical quality.

In the future, it is planned to consider the urban areas in the analysis and also use the VH polarized TerraSAR-X image to investigate the impacts of different polarizations in the fusion outputs. In addition to that, image classification processes will be applied to evaluate the impacts of fusion algorithms over land use/cover thematic map production.

\section{REFERENCES}

Abdikan, S., Sanli F.B., 2012. Comparison of different fusion algorithms in urban and agricultural areas using SAR (PALSAR and RADARSAT) and optical (SPOT) images. Boletim de Ciências Geodésicas 18, pp. 509-531.

Abdikan, S., Sanli F.B., Sunar, F., Ehlers, M., 2012. A comparative data-fusion analysis of multi-sensor satellite images. International Journal of Digital Earth, DOI:10.1080/17538947.2012.748846.

Abdikan, S., Sanli FB., Filiz B.B., Goksel, C., 2008. Fusion of SAR images (PALSAR and RADARSAT-1) with multispectral spot image: a comparative analysis of resulting images. In: The International Archives of the Photogrammetry Remote Sensing and Spatial Information Sciences 37 (B7), 1197-1202.

Berger, C., Hese, Soren, Schmullius, C., 2010. Fusion of high resolution SAR data and multispectral imagery at pixel level-A statistical comparison. In: Joint SIG Workshop, Urban- 3D Radar - Thermal Remote Sensing and Developing Countries Ghent, 22-24 September 2010, pp. 245-268

Ehlers M., Klonus, S., Åstrand, P.J., Rosso, P., 2010. Multisensor image fusion for pansharpening in remote sensing. International Journal of Image and Data Fusion 1, pp. 25-45.

Güngör, O., Yıldırım. D., 2012. A novel image fusion method using IKONOS satellite images. Journal of Geodesy and Geoinformation 1, pp. 27-34.

Hong G., Zhang, Y., Mercer, B., 2009. A Wavelet and IHS Integration Method to Fuse High Resolution SAR with Moderate Resolution Multispectral Images. Photogrammetric Engineering \& Remote Sensing, 75(10), pp. 1213-1223.

Jalan, S., Sokhi, B.S., 2012, Comparison of different pansharpening methods for spectral characteristic preservation: multi-temporal CARTOSAT-1 and IRS-P6 LISS-IV imagery. International Journal of Remote Sensing, 33(18), pp. 56295643.

Klous, S., 2008, Comparison of pansharpening algorithms for combining radar and multispectral data. In: The International Archives of the Photogrammetry Remote Sensing and Spatial Information Sciences 37 (B6), pp.189-194.

Lu, D., Li, G., Moran, E., Dutra, L., Batistella, M., 2011. A Comparison of Multisensor Integration Methods for Land Cover Classification in the Brazilian Amazon. GIScience \& Remote Sensing 48, pp. 345-370.

\section{PCI Geomatica, 2012. Geomatica Help Menu}

Wang, Z., Bovik A.C., 2002. A Universal Image Quality Index. IEEE Signal Processing Letters 9, pp. 81-84.

Witharana, C., Civco, D.L., Meyer, T.H., 2013. Evaluation of pansharpening algorithms in support of earth observation based rapid-mapping workflows. Applied Geography 37, pp. 63-87.

Zhang, Y., 2009. Adjustable SAR-MS fusion (ASMF) for diverse geo-spatial applications. Joint Urban Remote Sensing Event, Shanghai, May 20-22. 